\title{
Ureteral iliac artery fistula in idiopathic retroperitoneal fibrosis: A case report
}

\author{
Eugenio Di Grazia ${ }^{1}$, Tiziana La Malfa ${ }^{2}$, Gherardo Gasso ${ }^{3}$ \\ ${ }^{1}$ U.O.C. di Urologia - ARNAS Garibaldi-Nesima, Catania, Italy; \\ ${ }^{2}$ Laboratorio Analisi - Casa di Cura Mater Dei, Catania, Italy; \\ ${ }^{3}$ U.O.C. di Chirurgia Vascolare - ARNAS Garibaldi-Nesima, Catania, Italy.
}

\begin{abstract}
Summary Ureter-arterial fistula (UAF) is an uncommon condition. The presentation is usually a life-threatening intermittent massive gross hematuria and the diagnosis is still a challenge for urologist. Idiopathic Retroperitoneal fibrosis (IRF) is a condition of unknown etiology characterized by a highly fibrotic retroperitoneal mass that frequently causes ureteral obstruction. To our knowledge we report the first case describing the UAF in a patient suffering from IRF. We hypothesize that inflammation and fibrosis resulted in fixation of the ureter to the adjacent artery causing a fistulous path. UAF was managed by deploying a $10 \times 59$ $\mathrm{mm}$ endo-graft at the intersection of common iliac artery bifurcation with the right ureter. Post treatment course was uneventful.
\end{abstract}

KEY WORDS: Idiopathic retroperitoneal fibrosis; Stent; Ureter; Uretero-arterial fistula.

Submitted 25 August 2019; Accepted 21 September 2019

\begin{abstract}
INTRODUCTION
Ureter arterial fistula (UAF) is an uncommon condition and about 150 cases are described in the literature. UAF can develop with aorta, common iliac artery, external iliac artery and hypogastric artery.

Fistulas between ureter and common or external iliac artery are usually associated with a history of pelvic surgery, pelvic irradiation, chronic ureteral stenting and vascular disease $(1,2)$. The presentation is usually a lifethreatening massive gross hematuria with acute anemia and the diagnosis is still a challenge for urologist, because of misleading cross-sectional imaging, ureteral contrast-enhanced studies and endoscopy to assess the fistula.

Digital subtracted angiography and vascular treatment are considered to be the best tools for the ureteric-arterial fistula assessment and management (2).

Retroperitoneal fibrosis (RPF) is a condition of variable etiology characterized by a highly fibrotic retroperitoneal mass that frequently causes ureteral obstruction. RPF encompasses the idiopathic form (IRF) (> 75\% of the cases) and secondary forms, which include cases secondary to malignancies, infections, drugs, radiotherapy, or other conditions. To our knowledge we report the first case describing the UAF in a patient with IRF.
\end{abstract}

\section{Case reports}

A 73-year-old male patient with gross hematuria was admitted to the emergency room. Anamnestic evaluation revealed a history of multiple aortocoronary bypass, bilateral inguinal hernioplastic, hypertension treated with antihypertensives and anticoagulants and idiopathic retroperitoneal fibrosis assessed one year earlier for obstructive renal failure. The patient was on treatment with Prednisone and bilateral ureteral stents. Patient was clinically stable, hemoglobin was $10 \mathrm{mg} / \mathrm{dl}$ and other blood chemistry parameters were normal. CT scan showed slight enlargement of the aorta associated with retroperitoneal fibrosis involving the abdominal aorta and the common iliac arteries. Blood clots were present in the right pyelocaliceal system, along the right ureter and in the bladder. Ureteral stents were in place. Cystoscopy and a thorough endoscopic evaluation of the right upper urinary tract revealed no existing disease. The following days new episodes of gross-hematuria with right flank pain requiring blood transfusions occurred. After hemodynamic stabilization the patient was urgently subjected to digital angiography. After removal of the double J stent that was replaced by a guidewire, selective arteriography of the common iliac artery and its branches did not show any fistulous tract with the right ureter. It was therefore decided to place a $10 \times 59 \mathrm{~mm}$ endovascular prosthesis at the intersection of common iliac artery bifurcation with the right ureter on the basis of previous CT scan evaluation of iliac artery ureteral crossing (Figure 1). Ureteral 6 x 26 stents were deployed again bilaterally in the upper urinary tract. The procedure was uneventful and the patient was discharged five days later without presenting new episodes of blood loss. One month later duplex sonographic evaluation of common iliac and external arteries showed normal blood flow and normal flowmetric indices.

UAF is classified into primary (15\%) and secondary (85\%) type on the basis of its cause. Primary causes are natural disease processes of the arterial system such as aneurysms, vascular malformations, or aberrant vessels that erode into the ureter. Secondary causes are pelvic surgery $(89 \%)$ combined with radiation (43\%) and with ureteral stent placement $(67 \%)$ leading to inflammation and fibrosis that, in turn, result in fixation of the ureter to the adjacent artery. High arterial pressure is transmit- 
Figure 1.

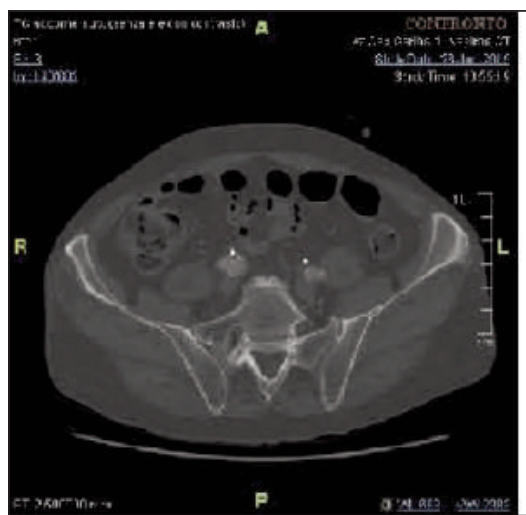

CT scan showing the intersection of common iliac artery bifurcation with the right ureter where the fistula path is supposed to be.

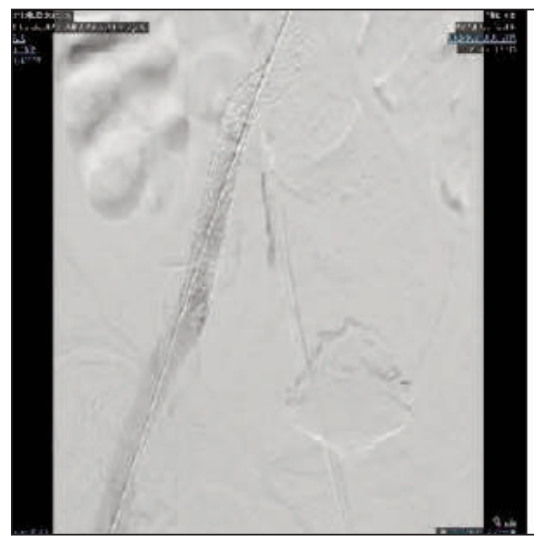

Endovascular prosthesis deployment at the intersection of common iliac artery bifurcation with the right ureter.

ted to the juxtaposed arterial and ureteral walls and supposed to determine pressure necrosis and fistula formation. The pressure head of arterial pulsion on a weakened arterial wall against a scarred and fibrotic stented ureter may facilitate fistula formation. Idiopathic RPF is a rare disease, with an estimated incidence of 0.1-1.3 cases $/ 100,000$ persons per year, and a prevalence of 1.4 cases/100,000 inhabitants (3). Idiopathic RPF disease usually involves the adventitia of the abdominal aorta and the iliac arteries and the surrounding retroperitoneum, and histologically shows a mixture of fibrous tissue and chronic inflammation involving the ureters.
The fibrous tissue comprises an extracellular matrix composed of type I collagen fibers organized in thick irregular bundles. Plasma cells account for a significant proportion of the inflammatory cells. Angiographic findings of UAF can vary from dramatic extravasation or a pseudoaneurysm to a subtle irregularity or intimal defect.

In approximately one third of the patients with UAF angiography may not reveal abnormal findings, making UAF challenging to observe. Another important issue is that the onset of hematuria is usually spontaneous but can be incited by initiation of anticoagulation or ureteral stent manipulation. Multiple options exist to treat UAF using the endovascular approach including stent-graft exclusion of the fistula and coil embolization of the affected artery. If stent-graft placement involves the iliac artery bifurcation, embolization of the ipsilateral internal iliac artery with coils or a vascular plug is considered to prevent retrograde feeding of the fistula.

\section{Conclusions}

IRF and chronic ureteral stenting may determine a UAF with life-threatning severe bleeding. Diagnosis is a challenge for Urologist and Radiologist. Minimally invasive angiographic management is recommended for high success and low complication rates. evaluation showing the prostheris and the patency of iliac arteries.

\section{REFERENCES}

1. Pillai AK, Anderson ME, Reddick MA, et al. Ureteroarterial fistula: diagnosis and and management. AJR Am J Roentgenol. 2015; 204:W592-W598

2. Mahlknecht A, Bizzotto L, Gamper C, Wieser A. A rare complication of ureteral stenting: Case report of a uretero-arterial fistula and revision of the literature. Arch Ital Urol Androl. 2018; 90:215-21.

3. Rafiei A, Weber TA, Kongnyuy M, Ordorica R. Bilateral ureteraliliac artery fistula in a patient with chronic indwelling ureteral stents: a case report and review. Case Rep Urol. 2015; 826760.

4. Vaglio A, Maritati F. Idiopathic Retroperitoneal Fibrosis J Am Soc Nephrol. 2016; 27:1880-1889.

\section{Correspondence}

Eugenio Di Grazia, MD (Corresponding Author)

e.digrazia@ao-garibaldi.ct.it

U.O.C. di Urologia - ARNAS Garibaldi-Nesima, Catania (Italy)

Tiziana La Malfa, MD

Laboratorio Analisi - Casa di Cura Mater Dei, Catania (Italy)

Gherardo Gasso, MD

U.O.C. di Chirurgia Vascolare - ARNAS Garibaldi-Nesima, Catania (Italy) 